\title{
ASTROMETRY WITH SCHMIDT TELESCOPES
}

\author{
C A Murray \\ Roya1 Greenwich Observatory
}

\section{INTRODUCTION}

Photographic astrometry, including work with Schmidt telescopes, can be divided into two main fields, (i) the measurement of positions of objects relative to a reference frame of stars with known celestial coordinates, and (ii) the measurement of relative proper motions and trigonometric parallaxes from a series of plates taken on the same field. The former demands a knowledge of the absolute transformation between angles on the sky and measurements on a plate, whereas in the latter we are only interested in differential transformations from plate to plate. The potential value of Schmidt telescopes for both these fields of astrometry lies in the large area of sky and range of magnitude which can be imaged on a single plate. The former advantage is however, to some extent offset by the curvature of the focal surface which means that, in order to utilize the full field the plates must be constrained to the form of the focal surface during exposure.

Some ten years ago, a conference with a title very similar to that of this colloquium was held at Hamburg at a time when the two large Schmidt telescopes were being constructed at Siding Spring and La Silla. Since that time the surveys which have been and are being produced by these two telescopes in the southern hemisphere together with the earlier Palomar Survey (POSS) of the sky north of - $30^{\circ}$ declination, have become essential sources of data for obtaining positions of celestial objects. Many astronomers now use these published atlases in the form of glass, film or even paper copies for the routine derivation of positions for identification purposes, although usually confining their measurements to a small area of a field. Hunstead (1974) has shown that a positional accuracy of $0: 3$ can be achieved using paper prints of the POSS.

Smaller Schmidt telescopes, too, are are now regularly used for positional astrometry. In particular we may mention the work on Pluto here at Asiago (e.g. Barbieri et al. 1979) and optical positions of 
radio sources both here (Barbieri et al. 1972) and at Cambridge (Argue et al. 1979).

\section{THE STELLAR REFERENCE FRAME FOR SCHMIDT PLATES}

The main contribution to the error of a position derived from Schmidt plates at the present time arises from the inaccuracies in the catalogue positions of stars defining the reference frame. Since these are nearly always extrapolated from earlier observations, errors in catalogue proper motions produce a continual deterioration in the reference frame.

The AGK3 catalogue (Heckmann and Dieckvoss 1975) is now used almost universally as the source of reference stars in the northern hemisphere. The proper motions in AGK3 are based on observations made at about 1930 and 1959; the resulting error of a single star coordinate at the current epoch is about $\pm 0: 44$ and by the end of the century will amount to at least \pm 0.5 .

In the southern hemisphere the situation at the present time is much worse. The most readily available source of star positions is the SAO Catalogue (Smithsonian Institution 1966) which, in the southern hemisphere, is based primarily on photographic zone catalogues observed at Yale (Johannesburg) and at the Cape prior to 1950, with proper motions obtained from comparison with earlier meridian observations. The reference frame in the southern hemisphere will however soon be greatly improved when work on the Second Cape Photographic Catalogue (CPC2) is completed. The 5800 plates taken for this project, between 1962 and 1970 are being measured on the GALAXY automatic measuring machine at RGO. Although more than seventy five per cent of the plates have now been measured, completion of the project is now in jeopardy because of the recent decision of the Science and Engineering Research Council to withdraw support for GALAXY at the end of March 1984. A first instalment of CPC2, giving provisional positions at epoch 1962 with an average internal standard deviation of \pm 0.1 in each coordinate for 50,000 stars in the zone $-38^{\circ}$ to $-54^{\circ}$, is now available from RGO and through the Strasbourg Stellar Data Centre. Proper motions for more than 20,000 of these stars which are in the SAO Catalogue have also been redetermined. Measurement is complete for the more southerly declinations and provisional positions for these should be available within a year or so. The final positions will be obtained by a block adjustment (de Vegt and Ebner 1974) of the measurements on all the plates covering the whole southern hemisphere.

A further dramatic improvement in the reference frame over the whole sky will becom available early in the next decade, with the completion of the HIPPARCOS mission which will give positions, annual proper motions and trigonometric parallaxes with average mean error of \pm 0!002 for some 100,000 selected stars, and the associated TYCHO 
experiment which will give positions at mean epoch 1989 with accuracy of a few hundredths of an arc second for all stars brighter than about $B=$ 11 .

\section{GLOBAL REDUCTION OF A SCHMTDT PLATE}

The main distinction between Schmidt astrometry and what we may term 'flat field' astrometry, is that, in the former, distances measured along great circles over the spherical focal surface are proportional to the corresponding angular distances on the sky, whereas in the latter, linear distances in the focal plane measured from the tangential point are proportional to the tangents of the corresponding angles. To a high degree of approximation therefore, the difference between tangential coordinates and coordinates measured on a Schmidt plate can be described by a purely geometrical third order radial distortion with coefficient $1 / 3$ if all angles are measured in radians. Although in principle, because of spherical symmetry, a Schmidt plate has no unique origin, corresponding to the tangential point in flat field astrometry, in practice the simplest model of elastic deformation during exposure will be symmetrical about some neutral point. Shepherd (1953) has shown that the effect of such deformations for a glass plate can be expressed as a linear scale change and a small third order radial distortion. Radial distortions and even more complicated transformations can in principle be derived from the adjustment of measurements of reference stars to their catalogue position, but this is generally impractical because of the errors of the positions obtained from the main source catalogues, AGK3 or SAO.

Special investigations of the astrometric performance of Schmidt telescopes are therefore carried out in particular fields in which relative positons with high positional accuracy are available, such as the region of $\alpha$ Persei (Heckmann et al. 1956), Praesepe (Russell 1983) and the Pleiades (van Leeuwen: to be published). The overlapping plate technique could also be used.

Anderson (1971) studied the performance of the Brorfelde Schmidt telescope using plates taken on the $\alpha$ Persei field for which the estimated mean error for one coordinate was $\pm 0 " 115$. He showed that the radial distortion relative to tangential coordinates could be represented quite adequately by the geometrical term, with coefficient $1 / 3$, over the field of $5: 3$ diameter. Allowing for the estimated error of star positions he found the external mean error of one position measured on one plate to be about $\pm 0: 20$, compared with the internal error of $\pm 0: 14$; the difference between these two was attributed to residual plate distortions and emulsion shifts as well as unmodelled optical aberrations.

In order to examine plate effects Bru and Lacroute (1973) proposed an experiment in which a reseau of reference marks was photographed on 
to a Schmidt plate in its constrained (spherical) state. By intercomparing the measurements of these reference marks on a number of plates taken with the Palomar Schmidt, also on the $\alpha$ Persei field, Fresneau (1978) was able to show that the effects of plate errors were less than one micron, compared with his measurement error of $1.7 \mu$. Fresneau further studied the error covariance function of the residuals from an empirical 20 parameter plate constant solution and found significant correlations between residuals extending to separations between stars of up to $5 \mathrm{~cm}$ (about $1^{\circ}$ ). He was able to improve the residuals by $\pm 1.7 \mu$ by applying a statistical smoothing process.

\section{ATMOSPHERIC DISPERSION}

A major potential error source in hight precision astrometry is the variation of atmospheric refractivity with star colour. In classical astrometry with photographic refractors this has never been too much of a problem since the objective itself filters out most of the ultraviolet light. In the case of a Schmidt, or for that matter any reflecting telescope, colour dependence of refraction on unfiltered plates can be large. Elsewhere (Murray and Corben 1979) I have shown plots of the average constant of refraction for various emulsions over a limited range of star colours; these were calculated by convolving the wavelength dependent constant of refraction for standard atmospheric conditions given by Allen (1973) with the
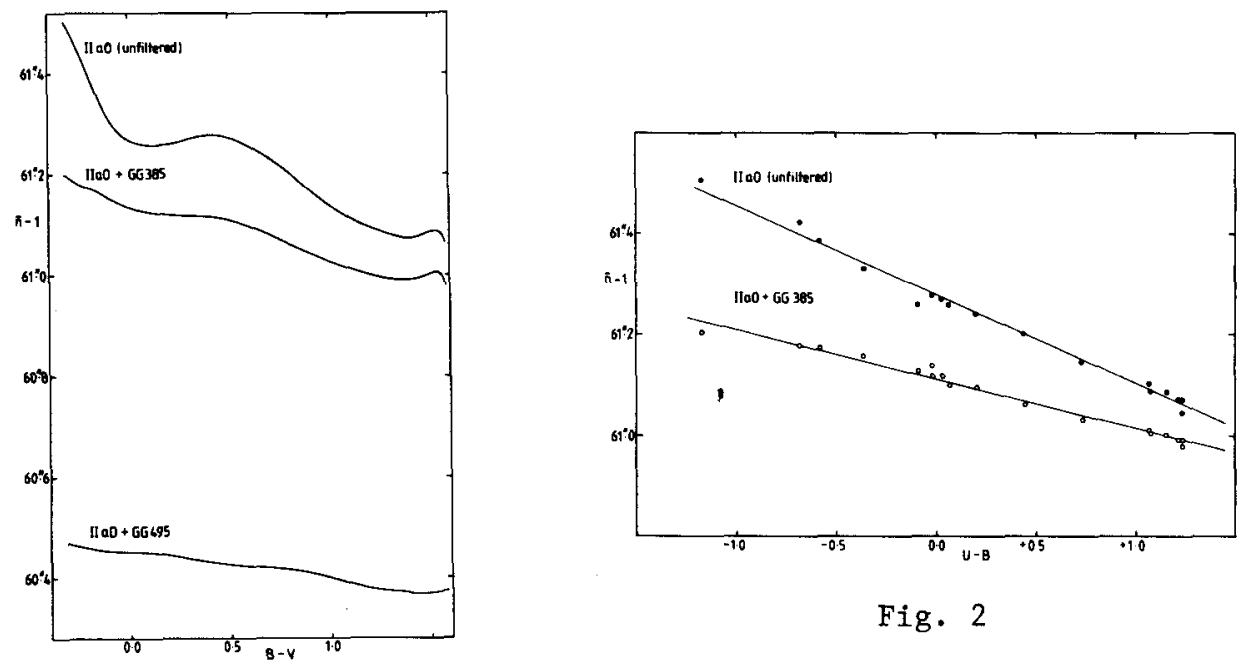

Fig. 2

Fig. 1 
transmissivity of the atmosphere and telescope, the emulsion sensitivity and stellar energy distributions. We have recently recomputed the refractivity in a slightly more rigorous fashion. Writing the refractive index for wavelength $\lambda$ in the form

$$
\mathrm{n}(\lambda)=1+\beta_{\mathrm{d}}(\lambda) \rho+\left(\beta_{\mathrm{w}}\left(\lambda,-\beta_{\mathrm{d}}(\lambda)\right) \rho_{\mathrm{w}}\right.
$$

where $\rho$ is the total density of air and $\rho_{w}$ the water vapour density, we have computed effective values $\bar{\beta}_{d}, \bar{\beta}_{w}-\bar{\beta}_{d}$ for various sensitivities and stellar energies for unreddened class $V$ stars taken from the tables of Straizys and Sviderskiene (1973). Values of the effective refractivity $\bar{n}-1$, expressed in arc seconds for unit afr mass and the same standard atmospheric conditions as in Allen's table (1oc. cit.) are shown as a function of $B-V$ in Fig 1 . The very marked dip in the curve for the IIaO emulsion corresponding to the Balmer discontinuity is obvious, and suggests that the refractivity in this case is much more conveniently expressed in terms of U-B. From Fig 2 we see that in fact to a high approximation the refractivity is linear with $U-B$. Least squares fits to the individual points which are shown in the figure are:-

$$
\begin{aligned}
& \text { IIa0 (unfiltered) } \bar{n}-1=61: 281-0: 176(U-B) \\
& I I a 0+G G 385 \quad \bar{n}-1=61: 111-0: 098(U-B)
\end{aligned}
$$

The IIIaJ emulsion shows similar characteristics.

It should be emphasized that these data refer to the refractivity corresponding to absorption in one air mass; this is not quite the same as the constant of refraction at zenith distance $\theta_{0}=45^{\circ}$. of course the actual amount of refraction is approximately proportional to $\tan \theta_{0}$, so that relative displacements of up to one arc second between extreme red and blue stars at large zenith distance are quite possible.

\section{GALACTIC ASTROMETRY}

I would like to conclude this review by discussing astrometric programmes for the measurement of proper motions and parallaxes.

By far the most extensive and successful programme of this nature which has yet been carried out with a Schmidt telescope is the survey for stars with large proper motion by Luyten, using plates taken with the Palomar 48-inch Schmidt. With the exception of about 160 very crowded Milky Way fields, Luyten has blinked, either with his automatic scanner or by eye, all the Palomar survey fields north of $-33^{\circ}$ declination. Combining these new data with earlier surveys by himself and others he has now published a revised LHS Catalogue (Luyten 1979) containing all known stars with annual proper motions exceeding $0: 5$ and three volumes of the NLTT catalogues (Luyten 1979-80) containing data for all stars with annual proper motions estimated to exceed $0 ! 18$. The fourth volume of the NLTT Catalogue (Luyten 1980) covers the sky south 
of declination $-30^{\circ}$. It contains roughly only half the number of stars in the corresponding zone of the northern hemisphere. Apart from a special search to faint limits with the Palomar Schmidt around the south galactic cap, this fourth volume is based largely on his earlier compilations, LTT (Luyten 1957) and the Bruce Proper Motion Survey (Luyten 1963). There is a rich field to be harvested here when the current southern hemisphere surveys are ripe for repetition; Luyten himself started his Palomar survey on red sensitive plates, in 1962, some fifteen years after the first poss plates were taken. Maybe it is not too early now to plan reobservation of one or more of the southern hemisphere surveys; the first plates for the SRC ' $J$ ' and ESO ' $B$ ' surveys are already ten years old. Although for strict comparability with Luyten's work the extension to faint limits in the southern hemisphere should be based on the ESO red survey, this would delay proper motion measurements to near the end of the century.

A proper motion programme of rather a different character is being carried out at the Lohrmann Institute, Dresden with the aim of calibrating the proper motion systems of meridian circle catalogues (Böhme and Sandig 1979). Proper motions will be measured relative to extra galactic objects on plates taken with the $2 \mathrm{~m}$ Universal Reflecting Telescope of the Karl-Schwarzschild Observatory, Tautenburg, in its Schmidt mode. Two exposures are obtained on each plate covering a $391 \mathrm{x}$ 3.1 square field; Magnitude compensation is provided by means of a subbeam prism with reduction of 3.9 magnitude.

While the detection of interesting stars with high proper motions may be described as the cream, there is much solid meat to be extracted from a study in depth of the large amount of astrometric and photometric data which can be obtained from plates taken on a single field.

A few years ago, the first results of an extensive study of a field near the south galactic cap were reported (Murray and Corben 1979). The plates for this programme were taken with the UK Schmidt telescope and measured on GALAXY at RGO. From an analysis of about fifty plates taken over three years we were able to obtain proper motions and parallaxes with standard errors of \pm 0 :013 and $\pm 0: 018$ respectively for stars brighter than $B=14$. These parallaxes were no worse than those obtained in the routine parallax program of the least accurate of the long focus refractors. For the first time it has been possible to determine the statistical distribution of proper motions and parallaxes, as functions of colour, of a sample of stars limited only by apparent magnitude. For various reasons further progress has been delayed; however, more than 100 plates on this field have now been measured giving data for about 16000 stars brighter than $B=18$. In addition nearly fifty plates have been obtained on two new fields, the MortonTritton field at $\alpha_{1950}=22^{\mathrm{h}} 03^{\mathrm{m}}, \delta_{1950}=-18^{\circ} 55^{\prime}$ and Pickering's E8

region at $\alpha_{1950}=20^{\mathrm{h}} 05^{\mathrm{m}} \cdot \delta_{1950}=-44^{\circ} 59^{\prime}$ 
but future work on these fields is likely to be delayed because of the closure of GALAXY.

It is now nearly eighty years since Kapteyn proposed his programme of Selected Areas. This was a far sighted project from which most of our present ideas about the distribution of stars in the Galaxy has been derived. The main astrometric contributions to the programme have been the measurement of proper motions which have formed the basis for studies of statistical parallaxes and velocity distributions of stars out to several hundred parsecs from the Sun. Similar programmes to fainter magnitudes and larger samples using Schmidt plates will extend such studies to the population, space distribution and kinematics of stars to distances of several kiloparsecs.

One of Kapteyn's objectives was the systematic measurement of trigonometric parallaxes in each area, but for reasons which are obvious to us, nothing of value came from this aspect of the programme. With Schmidt astrometry we can now remedy this. It would for example be relatively straightforward to test the completeness of Gliese's catalogue of nearby stars, in which the average surface density is rather less than two per field of the UK or Palomar Schmidt telescopes. By analysing smaller parallaxes in a large number of fields it should be possible for the first time to obtain direct evidence for the true density and luminosity distribution in the solar neighbourhood, completely unbiased by the proper motion selection effects which have bedevilled earlier work in this field.

The availability of Schmidt telescopes, particularly the large ones, and facilities for accurate automatic plate measurement provide a great opportunity for astronomers today to pursue astrometric programmes which will make fundamental contributions to our knowledge of our Galaxy.

\section{REFERENCES}

Allen, C.W. 1973. Astrophysical Quantities 3rd edition. (London: Athlone press), p 125

Andersen, J. 1971. Astron. \& Astrophys. 13, 40.

Argue, A.N., Clements, E.D., Harvey, G.M. and Murray, C.A. 1979 IAU Colloquium No 48, eds. F.V. Prochazka and R. H. Tucker (University of Vienna) p 156.

Barbieri, C., Capaccioli, M., Ganz, R. and Pinto, G. 1972 Astron. J., $77,444$.

Barbieri, C., Benacchio, L., Capaccioli, M. and Pinto, G. 1979 Astron. J., 84,1890 .

Böhme, D. and Sandig, H. U. 1979 IAU Colloquium No 48, eds. F.V. Prochazka and R. H. Tucker (University of Vienna) p 535.

Bru, P. and Lacroute, P. 1972. The role of Schmidt Telescopes in Astronomy, ed. U. Haug (Hamburg-Bergedorf) p 45.

de Vegt, Chr. and Ebner, H. 1974. Mon. Not. R. Astron. Soc. 167, 189.

Fresneau, A. 1978. Astron. J., 83, 406. 
Heckmann, 0., Dieckvoss, W. and Kox, H. 1956. Astr. Nachr. 283, 109. Heckmann, 0. and Dieckvoss, W. 1975. AGK3 Star Catalogue of Positions and Proper Motions North of $-2: 5$ Declination (Hamburg-Bergedorf)

Hunstead, R.W. 1974 IAU Symposium No. 61, eds W. Gliese, C.A. Murray, and R.H. Tucker (Dordrecht: Reidel) p 175.

Luyten, W.J. 1957. A Catalogue of 9867 Stars in the Southern Hemisphere with Proper Motions Exceeding 0:2 annually (Minneapolis, Minnesota: Lund Press).

Luyten, W.J. 1963. Bruce Proper Motion Survey - The General Catalogue (Minneapolis, Minnesota).

Luyten, W.J. 1979-80. New Luyten Two Tenths Catalogue (University of Minnesota).

Murray, C.A. and Corben, P.M. 1979. Mon. Not. R. Astron. Soc. 187, 723.

Russe11, J.L. 1983. IAU Colloguium No. 76 (in press). Shepherd, W.M., 1953 Mon. Not. R. Astron. Soc. 113, 450.

Straizys, V. and Sviderskiene, Z. 1973. Bull. Vilnius Astron. Obs. No. 35. 